http://dx.doi.org/10.5007/2175-7968.2013v2n32p141

\title{
DENSIDADE DE INFORMAÇÃO, COMPLEXIDADE FONOLÓGICA E SUAS IMPLICAÇÕES PARA A ORGA- NIZAÇÃO DE GLOSSÁRIOS DE TERMOS TÉCNICOS DA LÍNGUA DE SINAIS BRASILEIRA
}

\author{
Janine Soares de Oliveira \\ Universidade Federal de Santa Catarina \\ janinemat@gmail.com \\ Markus Johannes Weininger \\ Universidade Federal de Santa Catarina \\ markus@cce.ufsc.br
}

\begin{abstract}
Resumo: Nesse artigo apresentam-se resultados parciais obtidos na análise de unidades terminológicas em Libras. A investigação foi motivada pela necessidade de ordenação dos sinais apresentados no Glossário do Curso Letras-Libras segundo os aspectos visuais da língua de sinais. Procurando atender às especificidades visuais-espaciais da Libras, a proposta inicial consistiu em ordenar o banco de dados segundo os parâmetros Configuração de Mão e Localização do Sinal. A complexidade dessa tarefa de ordenação evidenciou a necessidade de mapear a estrutura fonológica e morfológica das unidades lexicais que constituem o Glossário a fim de identificar quais seriam os filtros de busca mais eficazes a serem utilizados no software - com relação aos níveis e subníveis - para ordenação do sistema. A análise proposta mostrou vários aspectos que necessitam de aprofundamento principalmente com relação à definição de filtros de busca que atendam ao consulente surdo. No presente texto serão apresentados resultados parciais da análise destacando-se o mapeamento dos locais de realização do sinal mais recorrentes, bem como a análise do aspecto 'movimento' nas unidades terminológicas, além de resultados obtidos pela análise comparativa entre os dados do Glossário Letras-Libras e dados de língua geral. De acordo com a esperada densidade informacional mais
\end{abstract}


elevada da linguagem técnica (cf. Koch \& Oesterreicher, 1994), e pela iconicidade funcional postulada por Givón (1995), os termos técnicos em Libras pela sua maior complexidade de conteúdo mostraram uma maior complexidade fonológica pela sua composição (predominância de sinais bimanuais) e realização (todos com movimento) fazendo com que esses critérios não sejam adequados como filtros de refinamento de busca ou categorias de ordenação de um glossário.

Palavras-chave: Densidade de informação. Fonologia da libras. Lexicografia da libras.

\title{
INFORMATIONAL DENSITY, PHONOLOGICAL COM- PLEXITY AND THEIR IMPLICATIONS FOR ORGANI- ZING A GLOSSARY OF TECHNICAL TERMS IN BRAZI- LIAN SIGN LANGUAGE
}

\begin{abstract}
In this paper we present partial results obtained through the analysis of technical terms in Libras. The present research was inspired by the need to of the Letras-Libras undergraduate program at UFSC according to visual aspects of a signed language. Trying to match the specific visual and spatial features of Libras, the initial proposal was to organize the glossary database according to the parameters of handshape and sign location. The complexity of this task evidenced the need for a mapping of the phonological and morphological structure of the lexical items that compose the glossary that could be used as more efficient filters for the retrieval of items and for ordering the entries. The proposed analysis showed several aspects that require a more thorough inquiry especially due to the need presenting filters that match deaf users needs. This text shows some partial results emphasizing the mapping of sign location as well as of movement in terminological units and results of a comparison between out data from the Letras-Libras glossary with general libras data. Due to the expected higher information density of technical language (cf. Koch \& Oesterreicher, 1994) and the functional iconicity postulated by Givón (1995), the technical terms in Libras showed a higher phonological complexity in composition (clear majority of signs is bimanual) and realization (all with movement) resulting in the need to use other categories for filtering a refine search or for ordering the glossary.

Keywords: Information density. Libras Phonology. Libras Lexicography.
\end{abstract}




\section{Introdução}

Esse artigo apresenta resultados parciais obtidos através de análise fonológico-morfológica de unidades terminológicas em Libras. Essa investigação foi motivada pela participação em projeto de desenvolvimento de glossário do curso Letras-Libras - graduação, oferecida na modalidade de educação à distância sob coordenação da Universidade Federal de Santa Catarina através da parceria com outras 9 instituições de ensino ${ }^{1}$.

Todo material produzido pelos professores-autores para constituir o texto-base de cada disciplina do curso foi traduzido por uma equipe de tradutores surdos e disponibilizado em Libras para os estudantes. $\mathrm{O}$ processo de tradução destes materiais desencadeou descobertas, transformações, discussões e reflexões que acarretaram o desenvolvimento terminológico que avança a cada dia. Ao ter acesso a espaços antes desconhecidos, a comunidade surda brasileira revive o fenômeno de nomeação das descobertas que se repete ao longo da história da humanidade.

Os termos coletados/propostos pela equipe de tradutores surdos do curso Letras-Libras foram disponibilizados no ambiente virtual do curso, inicialmente sendo recuperados/consultados somente através de busca em português, mas, logo percebeu-se a necessidade de melhorar a organização e recuperação dos dados mais eficaz atendendo às especificidades da língua de sinais.

Dadas às particularidades da Libras, foi necessário elaborar um sistema que permitisse recuperar dados do banco de dados respeitando suas características visuais e não apenas o acesso pela ordem alfabética em português como ainda acontece na maioria dos dicionários e glossários de línguas de sinais. A procura por filtros de busca mais adequados às especificidades da Libras e às necessidades dos usuários surdos que sabem Libras, mas precisam acessar o item em português e dos usuários ouvintes que estão apreendendo Libras foi fator determinante para o desenvolvimento da investigação referente à análise das unidades lexicais da Libras. 
A investigação no campo da ordenação de repertório lexicográfico em Libras além de levar em conta os aspectos dessa língua deve considerar as funções desse repertório. As estruturas terminológicas contidas no Glossário Letras-Libras buscam atender à função principal de comunicar em área de especialidade, devendo, portanto atender os requisitos exigidos para a formação de itens terminológicos.

$\mathrm{Na}$ comunicação especializada em áreas técnicas, comumente surgem alguns processos de lexicais e sintáticos típicos para ao mesmo tempo aumentar a precisão por um lado, através da terminologização de itens lexicais e a quantidade de informações veiculadas dentro de um texto. O segundo fenômeno inclui a diminuição de redundância em relação à comunicação cotidiana por um lado, e por outro, a necessidade de explicitar elementos contextuais que normalmente não necessitam ser explicitados na comunicação da linguagem geral.

Além dos aspectos funcionalistas e terminológicos, é relevante destacar a questão do registro da língua que passa do nível de informalidade da interação direta e presencial para o nível do registro formal em contexto de distância do interlocutor. Nesse caso, assemelha-se aos fenômenos que distinguem língua falada de língua escrita gerando a expectativa de densidade lexical maior nessa última modalidade - no caso da língua de sinais representada pelo vídeo contendo as unidades terminológicas. Sobre essa densidade lexical Eggins (2004) afirma:

To this point the differences we have noted between the language of spoken and written situations are no doubt quite familiar to you. It is important to appreciate that these linguistic differences are not accidental, but are the functional consequence (the reflex) of the situational differences in mode. However, there are two more linguistic features that are highly sensitive to mode variation: the degree or grammatical complexity, and the lexical density of the language chosen. (Eggins, 2004, p.93-94) ${ }^{2}$ 
A densidade lexical, medida com ajuda da razão de tipos e ocorrência de itens lexicais (type-token ratio) indica no nível textual o grau de redundância no texto. Silva e Babini (2011) exemplificam este conceito no seu artigo sobre material terminológico em inglês. A densidade semântica e terminológica indica quantos elementos lexicais com função terminológica são usados num texto, cf. Guedes, Mollica e Leal (no prelo) e Sagi, Kaufmann e Clark (2009).

Homberger, Happ e Leuninger (2002) comparam a estrutura discursiva de línguas orais e línguas de sinais e chegam à conclusão que apesar da taxa de articulação (sinais/palavras por minuto) das línguas orais ser o dobro da das línguas de sinais, as duas acabam produzindo o mesmo conteúdo no mesmo tempo devido à capacidade das línguas de sinais de produzir sinais com maior densidade de informação. "The condensation of information is not achieved by the high-speed serialization of segments and morphemes but by the simultaneous output of auto-segmental phonological features and morphemes." " (ibid., p. 132). Ou seja, a maior densidade de informação pode ser reproduzida através de uma maior complexidade fonológica e morfológica. Já Givón (1995) lista (p.9) e depois (p. 10-22) especifica a premissa "clássica" da abordagem funcionalista de que a iconicidade funcional inclui a necessidade de uma maior complexidade linguística para reproduzir uma maior complexidade de conteúdo.

Partindo dos princípios funcionalistas supõe-se que as unidades terminológicas em Libras apresentem maior densidade de informação em comparação com unidades lexicais da língua geral. A fim de verificar essa hipótese serão apresentados dados de comparação entre a análise realizada no corpus de estudo e análise de repertório de língua geral realizada por Xavier (2006). 


\section{As ferramentas tecnológicas e a ordenação de repertórios lexicográficos}

O desenvolvimento tecnológico favorece o registro e divulgação das unidades terminológicas resultantes da expansão lexical experimentada pela Libras, pois tradutores surdos e não-surdos têm buscado compartilhar suas estratégias e decisões tradutórias através da internet, constituindo bancos de dados mais (ou menos) estruturados em diferentes áreas de especialidade. Como por exemplo, 'Terminologia da Política Brasileira em Libras'4, 'Libras e Saúde's, assim como diversos vídeos compartilhados no youtube, dentre os quais se destacam os vídeos do 'Projeto Glossário Científico em Língua Brasileira de Sinais ${ }^{\circ}$, entre outros.

Tais propostas confirmam a relação histórica estabelecida entre o campo da Terminologia e dos Estudos da Tradução, conforme observaram Krieger \& Finatto (2004):

"[...] a Terminologia mantém uma relação estreita e de longa data com a tradução técnico-científica, por isso a troca de experiências entre ambas é algo quase natural". (KRIEGER \& FINATTO, 2004, p. 177).

No entanto, apesar dos avanços tecnológicos recentes que permitem a produção e o compartilhamento de textos em língua de sinais através de ferramentas digitais online, observa-se carência no que se refere a bancos de dados sistematizados que sirvam como corpus de referência para análises dessas línguas.

As unidades terminológicas presentes nos materiais do curso Letras-Libras inicialmente só poderiam ser consultadas através de consulta à lista de termos exibida em língua portuguesa ou por agrupamentos das unidades de acordo com a(s) disciplina(s) a(s) qual(is) estava relacionada.

A tarefa de desenvolver um sistema para postagem, codificação, ordenação e recuperação da informação do banco de dados evidenciou a necessidade primeira de identificar os padrões de formação fonológica e morfológica das unidades terminológicas. 
Através de coleta e análise de outros repertórios lexicográficos de Língua de Sinais - destacam-se no quadro abaixo (quadro 1) os mais consultados pela equipe de tradutores - essa equipe definiu que os dados seriam organizados por Configuração de Mão (CM), local de realização do sinal e presença ou ausência de movimento.

\section{Quadro 1 - Síntese de Repertórios Lexicográficos Consultados}

\begin{tabular}{|c|c|c|c|c|c|c|}
\hline & 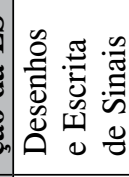 & $\sum_{0}^{\infty}$ & 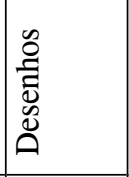 & $\frac{8}{0}$ & 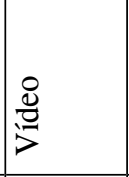 & $\frac{8}{0}$ \\
\hline 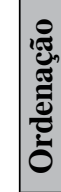 & 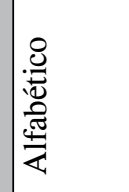 & 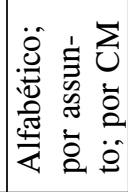 & 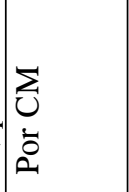 & 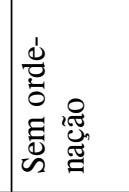 & 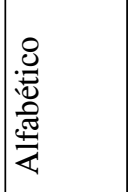 & 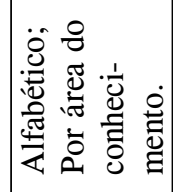 \\
\hline חٌ & 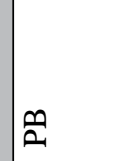 & $\stackrel{m}{2}$ & 兽 & 总 & 兽 & 总 \\
\hline 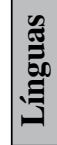 & $\cong$ & 番 & $\frac{\sqrt{2}}{4}$ & $\sqrt{2}$ & 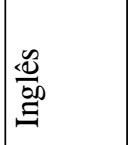 & 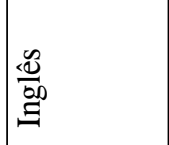 \\
\hline 爱 & 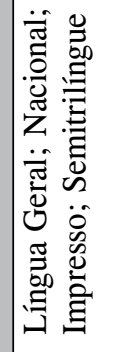 & 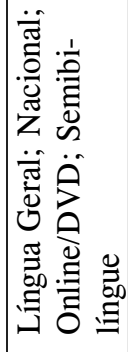 & 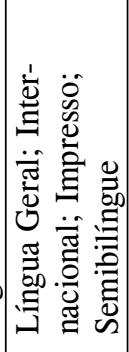 & 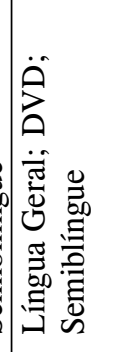 & 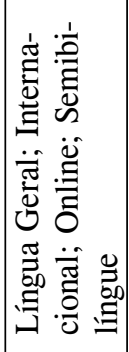 & 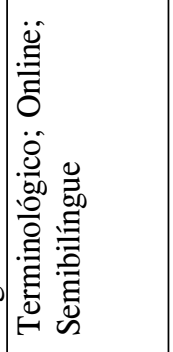 \\
\hline 总 & 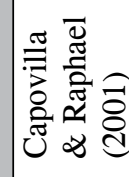 & 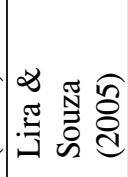 & 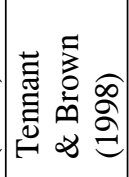 & 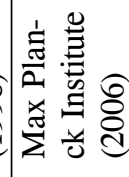 & 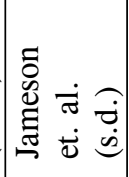 & 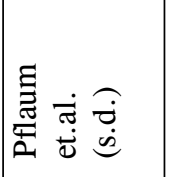 \\
\hline
\end{tabular}


Para codificar os dados nos filtros de busca, tomou-se como base a lista de Configurações de Mão conforme proposta do sistema de Escrita de Sinais ${ }^{7}$ conhecido como Sign Writing ${ }^{8}$. Sendo assim, o primeiro nível de ordenação dos dados seria o das configurações de mão agrupadas de acordo com o Alfabeto Internacional de Escrita de Sinais (ISWA), versão 2010․

\section{Metodologia}

Para a análise dos dados em vídeo utilizou-se o software ELAN (Eudico Linguistic Anotator) com o qual foi possível realizar a etiquetagem do corpus de estudo através de glosas da língua portuguesa. Além do ELAN utilizou-se também como instrumento de análise as imagens dos vídeos capturadas e segmentadas através do programa Free Video to JPG Converter. Pois, a especificidade da modalidade visual e espacial da língua de sinais exige a conjugação de recursos tecnológicos que contribuam para a observação dos fenômenos presentes na produção destas línguas.

Antes da análise mais aprofundada dos processos de formação de unidades léxicas especializadas em Libras, foi necessário realizar a coleta e classificação de repertórios terminológicos. A classificação foi realizada de acordo com a ficha de análise de repertórios lexicográficos proposta por Faria-do-Nascimento (2009). Em cada repertório foi realizada a contagem de vídeos e visualização de cada vídeo para coleta de unidades terminológicas. No presente texto serão apresentados resultados iniciais da análise dos vídeos em Libras produzidos para o Glossário Letras-Libras versão 2008-2010.

\section{Resultados parciais do corpus de estudo}

A seguir apresentam-se resultados parciais obtidos na análise de 100 vídeos do Glossário Letras-Libras versão 2008-2010. Na análise dos 100 vídeos identificou-se 132 unidades léxicas. A tabela 
(tabela 1) a seguir sintetiza os dados observados.

\begin{tabular}{|l|l|l|l|}
\hline Vídeos analisados & $\begin{array}{l}\text { Unidades léxicas } \\
\text { identificadas }\end{array}$ & $\begin{array}{l}\text { Quantidade } \\
\text { de Mãos }\end{array}$ & Movimento \\
\hline 100 & 132 & Uma mão $=15$ & \multirow{2}{*}{ Sim. Todos. } \\
\cline { 2 - 3 } & & Duas mãos $=117$ & \\
\hline
\end{tabular}

Tabela 1: Síntese dos vídeos analisados

Com base nas análises de repertórios lexicográficos em língua de sinais observou-se a recorrência da organização de repertórios segundo as configurações de mão da mão tradicionalmente chamada de dominante (Battison, 1978; Brentari, 1998). Porém, ao observar algumas unidades lexicais especializadas do glossário de Letras-Libras, ficou claro que a distinção entre "mão dominante/ mão de apoio" pode não ser suficiente para uma organização eficaz das unidades, pois, como observou-se na análise dos dados, acontecem casos onde a "mão de apoio" fornece traços importantes para a identificação de uma unidade lexical.

A seguir apresentam-se alguns exemplos que corroboram para a hipótese de existência de formantes produtivos para o processo derivacional. Como no caso dos sinais formados pela configuração de mão (figura 1) que ocorre de forma livre na realização da unidade lexical PALAVRA (figura 2).

Figura 1 - Configuração de Mão 9.33

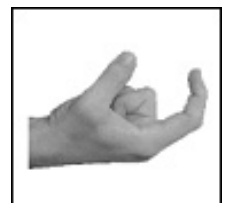

Fonte: SUTTON \& FROST, 2010. 


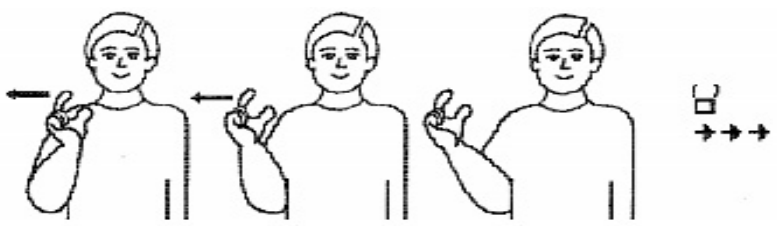

Fonte: CAPOVILLA \& RAPHAEL, 2001.

Observou-se a ocorrência desse mesmo formante (figura 1), nos sinais de LETRAMENTO, GLOSA, ESTRANGEIRIZAÇÃO, DESESTRANGEIRIZAÇÃO, NEOLOGISMO, COMPOSIÇÃO, JARGÃO, DERIVAÇÃO (figura 3), FLEXÃO (figura 4), MORFOLOGIA (figura 5) e MORFEMA (figura 6).

A análise da produção dos últimos quatro sinais (figuras 3, 4, 5 e 6) mostra que a configuração de mão 9.33 (figura 1) é marcada, como o primeiro movimento a surgir na realização do sinal.

\section{Figura 3 - Sinal DERIVAÇÃO}
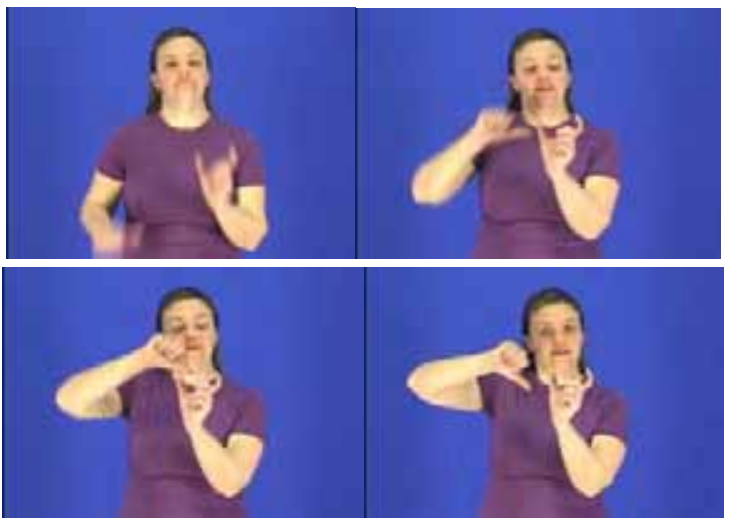
Figura 4 - Sinal FLEX̃̃o
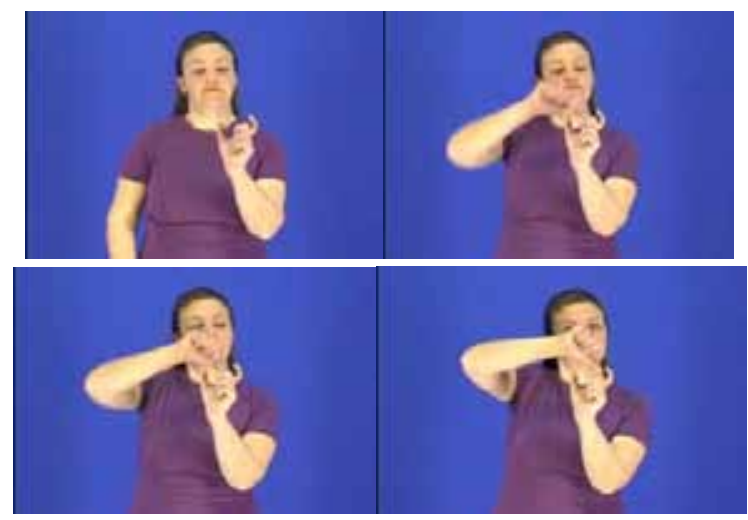

Figura 5 - Sinal MORFOLOGIA
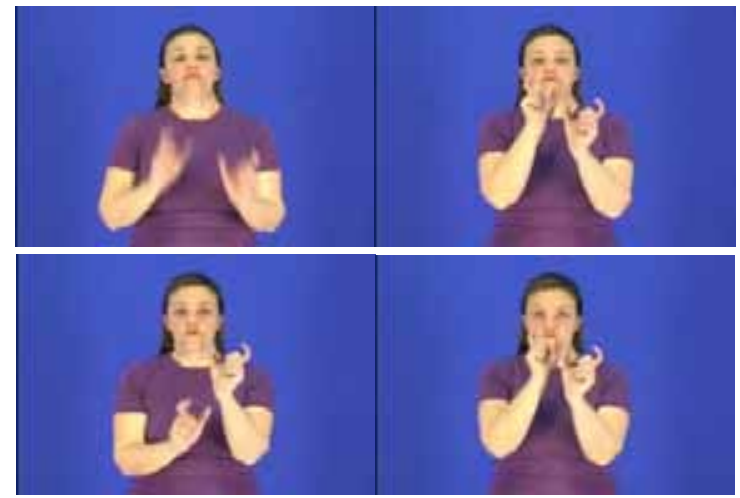

Figura 6 - Sinal MORFEMA

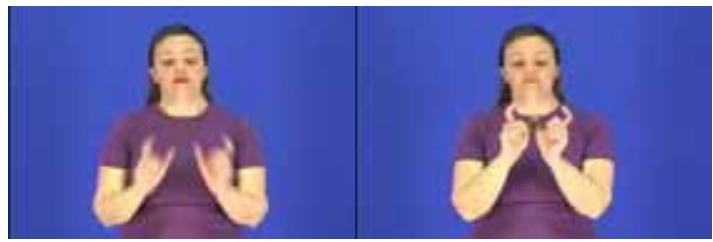




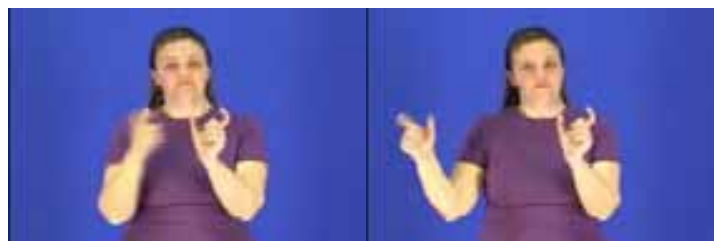

Apesar da tradição nas línguas de sinais com relação à mão dominante (Battison, 1978; Brentari, 1998) - direita para destros - e mão de apoio - esquerda para destros -observa-se que a presença desse formante (CM 9.33) na produção e compreensão dos sinais não se limita à função de apoio para a realização de sinais. Os dados observados contrapuseram a ideia de que a "mão não preferencial ou não dominante" atuaria somente como local de realização do sinal em caso de sinais assimétricos conforme afirmaram também Meir e colegas (2010):

However, according to some analyses, a different kind of simultaneous compounding is very widespread in sign languages. Signs in any sign language may be produced by one hand or by both hands. Two handed signs are symmetrical or asymmetrical. In symmetrical signs, the two hands have the same handshape and they articulate the same kind of movement at the same location (or at mirror locations on or near each side of the body). In asymmetrical signs, one hand (the preferred or dominant hand) articulates the sign, while the other hand (the nonpreferred or nondominant hand) is static and functions as the location of that sign. (MEIR et al., 2010, p. 11) ${ }^{10}$.

A importância do formante CM 9.33 (figura 1) foi comprovada nos testes de usabilidade realizados com a versão piloto do Glossário Letras-Libras. Na busca pelos sinais de NEOLOGISMO e JARGÃO, os consulentes optaram em filtrar os resultados por essa configuração de mão (figura 1) e não pela configuração de 
mão realizada pela mão supostamente dominante (figuras 7 e 8 , respectivamente).

Figura 7 - Configuração de Mão formante do sinal NEOLOGISMO

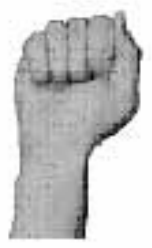

Fonte: SUTTON \& FROST, 2010.

Figura 8 - Configuração de Mão formante do sinal JARGÃO

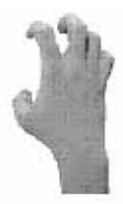

Fonte: SUTTON \& FROST, 2010.

Observando o exemplo do sinal de COMPOSIÇÃO (figura 9) ilustrado abaixo, pode-se formular como hipótese inicial para a formação do sinal a ideia de Reduplicação. Visto que COMPOSIÇÃO seria formada pela repetição do sinal PALAVRA (figura 2). Na língua portuguesa reduplicação pode ser definida como "um recurso morfológico em que uma mesma base é repetida duas ou mais vezes a fim de constituir um novo item léxico, geralmente pitoresco, por causa do inusitado procedimento de formação" (ALVES, 1990, p.70-71). 


\section{Figura 9 - Sinal COMPOSIÇÃo}
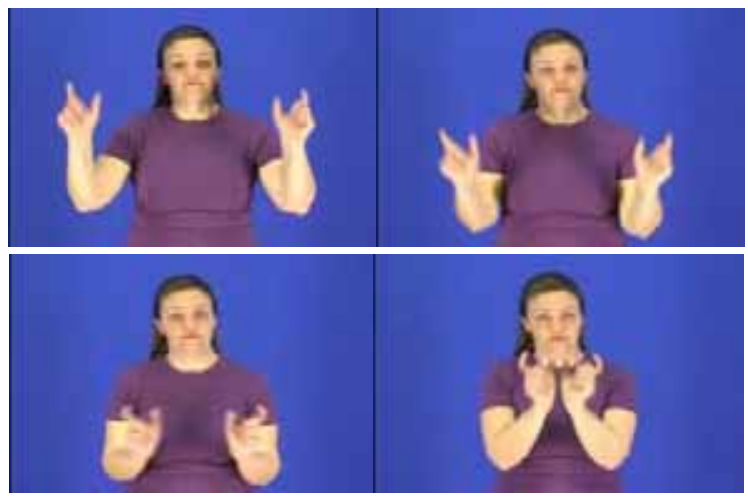

No entanto, as definições aplicadas às línguas orais - no caso o português - não devem ser aplicadas diretamente às línguas de sinais. Devido às especificidades dessa língua, em olhar mais apurado, observa-se que não se trata da simples repetição do sinal PALAVRA. Pois conforme, pode-se perceber nas figuras 2 e 9 e na transcrição em Escrita de Sinais de PALAVRA (figura 10.a) e COMPOSIÇÃO (figura 10.b) existe uma diferença significativa no parâmetro movimento - indicando pelas flechas - desses sinais.

\section{Figura 10 - Representação em Escrita de Sinais}

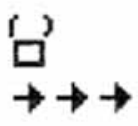

Figura 10.a: PALAVRA

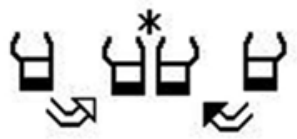

Figura 10.b: COMPOSIÇÃO

Tal comportamento fortalece a hipótese que o fenômeno morfológico composição conforme definido nas línguas orais é insuficiente para categorizar sinais de duas-partes em língua de sinais. 
Nas palavras de Liddell (2003):

Once created, compounds are subject to the pressures of historical change. [...] A typical ASL compound does not consist of two intact morphemes. This is because of the structural changes that take place as a result of compound formation, as well as historical changes subsequent to compound formation. Thus, a typical ASL sign that came into being by means of the compound formation rules is technically not a compound. I will continue to describe them as compounds, however, since the use of that term is widespread and there is no better term. (LIDDELLL, 2003, p. 16) ${ }^{11}$

Outro dado que relevante se refere à análise dos locais de realização dos sinais (gráfico 1).

\section{Local de realização}
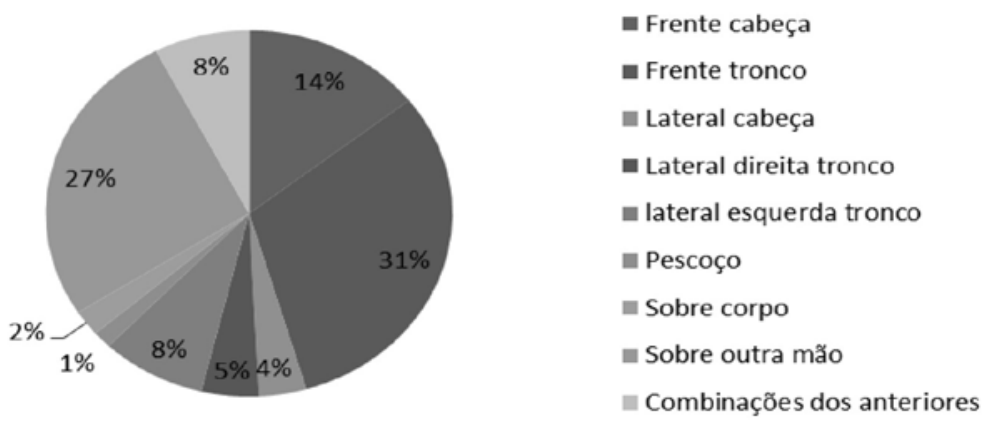

Gráfico 1: Local de realização dos sinais

Os resultados obtidos com a análise, ainda que iniciais, confirmam a necessidade de investigação aprofundada da formação de sinais terminológicos em Libras. 


\section{Análise comparativa entre dados do corpus de estudo e dados de léxico geral}

Xavier (2006) apresentou uma descrição da estrutura interna dos sinais da libras, de acordo com o modelo fonético-fonológico de $\mathrm{Li}$ ddell \& Johnson (1989). Para realizar essa descrição, o autor utilizou como corpus de estudo o dicionário de Capovilla \& Raphael (2001).

Segundo Xavier, apesar de o dicionário de Capovilla \& Raphael apresentar 4335 entradas lexicais, foram descritas em sua pesquisa 2274 formas. $\mathrm{O}$ autor explica que antes de fazer a análise propriamente dita, realizou alguns agrupamentos dos sinais, tendo categorizado 2274 entradas como 'sinais simples' e 861 como 'formas complexas', sendo estas últimas excluídas de sua análise.

$\mathrm{O}$ autor destacou ainda que dos 2274 sinais selecionados para a análise, 5 (cinco) eram realizados sem uso das mãos, apenas com expressões não-manuais, o que lhe deixou com um número final de 2269 unidades lexicais para verificar os fenômenos manuais de realização dos sinais.

Xavier definiu 7 categorias relacionadas à quantidade de mãos envolvidas na realização do sinal, existência/inexistência de movimento, existência/inexistência de movimento local, mudança ou não de configuração de mão durante a realização do sinal, existência/inexistência de contato, existência/inexistência de marcação não manual, existência/inexistência de reduplicação:

Como os modelos de análise apresentavam algumas diferenças, não foi possível comparar todos os fenômenos observados em cada pesquisa, no entanto já foi possível identificar alguns dados interessantes a partir dos resultados obtidos em um corpus de léxico geral e os dados de léxico especializado do corpus de estudo.

A seguir, apresentam-se alguns gráficos originados do cruzamento dos resultados das duas análises.

No gráfico 1 observa-se que foram encontrados mais sinais realizados com duas mãos no corpus de estudo (terminológico) do que no corpus de léxico geral. 


\section{Gráfico 1}

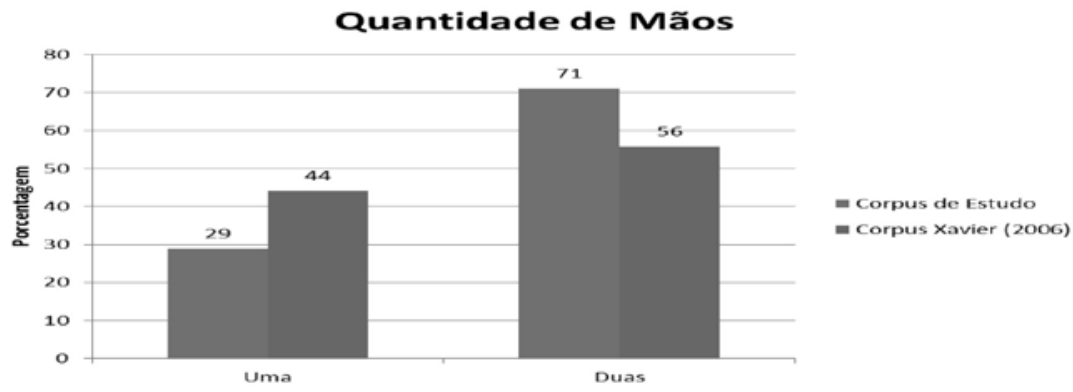

Conforme citado anteriormente, todos os sinais analisados no corpus de estudo apresentaram movimento. No gráfico 2 é possível visualizar esse resultado em relação aos dados obtidos por Xavier (2006).

\section{Gráfico 2}

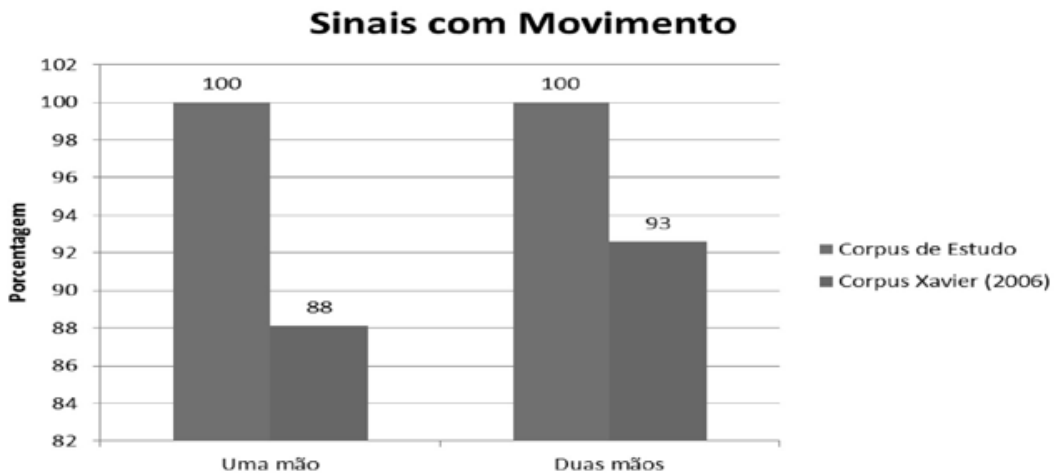


A análise comparativa entre dados do léxico geral e dados do léxico especializado confirmou para os critérios 'quantidade de mãos' e 'movimento' a hipótese maior complexidade e densidade de informação, ou seja, temos técnicos em libras usam movimento e as duas mãos maciçamente para codificar através do aumento de elementos fonológicos a carga semântica adicional de elementos terminológicos.

\section{Considerações finais}

A importância das duas mãos na realização de categorização dos sinais implicou a mudança no padrão de inserção e busca dos sinais no banco de dados do glossário Letras-Libras. Com a necessidade de disponibilizar a busca pela configuração de mão que o consulente julgar mais significativa (nem sempre a preferida ou dominante), o sistema deve oferecer resultados que apresentem a configuração de mão selecionada em quaisquer ocorrências (direita ou esquerda) não restringindo a busca à hierarquia de mão dominante.

$\mathrm{O}$ fato de todos os sinais analisados apresentarem movimento ocasionou o descarte dessa proposta de filtro visto que não haveria diferenciação mediante esse critério.

Observou-se que embora o "espaço neutro" fora subdividido em 4 - a saber, "frente cabeça", "lateral cabeça", "frente tronco", "lateral direita tronco" e "lateral esquerda tronco" - o resultado encontrado para "frente tronco" ainda foi significativo. Isso se dá mesmo com a inclusão da etiqueta "sobre outra mão", que também foi uma tentativa de diminuir os resultados para o filtro "frente tronco". Pois, a hipótese inicial era que a etiqueta mais geral "espaço neutro" apresentaria muitos resultados não sendo considerada como bom filtro para busca de sinais no sistema. Confirmada a hipótese que um número significativo de unidades lexicais especializadas receberia a etiqueta "frente tronco" considera-se imprescindível a inclusão de outros filtros de busca no sistema do novo 
Glossário Letras-Libras de modo a garantir uma quantidade de resultados adequada aos usuários do sistema. Visto que um filtro que apresente muitos resultados não atende ao seu objetivo principal que é o de permitir aos usuários identificar com rapidez a informação desejada baseado em elementos fonológicos e morfológicos visuais da libras.

\section{Notas}

1. A primeira turma ofertada em 2006 consistiu em parceria com 9 instituições de ensino superior para a habilitação Licenciatura em Letras-Libras. Em 2008, somaram-se mais 6 instituições as primeiras ampliando a oferta de vagas para Licenciatura e oferecendo novas turmas com habilitação para bacharelado em Letras-Libras.

2. Até este ponto, as diferenças que notamos entre a linguagem em situações faladas e escritas sem dúvida são bastante conhecidas. É importante apreciar que estas diferenças linguísticas não são acidentais, mas são da consequência funcional (o reflexo) das diferenças situacionais dos modos. Porém, há mais duas características linguísticas altamente sensíveis à variação do modo: o grau de complexidade gramatical e a densidade lexical da linguagem escolhida.

3. A condensação de informação não é alcançada mediante a serialização de segmentos e morfemas em alta velocidade, mas pela produção simultânea de morfemas e características fonológicas auto-segmentais.

4. Disponível em <http://politicaemlibras.blogspot.com/ $>$. Acesso em 01 fevereiro 2012.

5. Disponível em < http://librasesaude.blogspot.com/ > . Acesso em 01 fevereiro 2012. 
6. Alguns vídeos do projeto estão disponíveis no youtube e podem ser localizados buscando o nome do projeto no site.

7. A Escrita de Sinais é um sistema visual de registro das línguas de sinais. Os símbolos utilizados nesta escrita buscam expressar os movimentos, as formas das mãos, as expressões não-manuais e os pontos de articulação.

8. O sistema de escrita para línguas de sinais denominado SignWriting foi inventado há cerca de 30 anos por Valerie Sutton, que dirige o Deaf Action Commitee (DAC), uma organização sem fins lucrativos sediada em La Jolla, Califórnia, USA. Sua origem está em um sistema que a autora criou para notar os movimentos da dança. Conforme as publicações do DAC o sistema pode representar línguas de sinais de um modo gráfico esquemático que funciona como um sistema de escrita alfabético, em que as unidades gráficas fundamentais representam unidades gestuais fundamentais, suas propriedades e relações. O SignWriting pode registrar qualquer língua de sinais do mundo. (STUMPF, 2005, p.51-52)

9. "Existem dez grupos de símbolos para as mãos. As mãos são agrupadas de acordo com quais dedos são usados. Esses dez grupos são o começo da 'Sequência-de-Símbolos-SignWriting', que é a ordem dos símbolos usada para procurar sinais em dicionários escritos em SignWriting”. (STUMPF, 2005, p. 57)

10. Porém, de acordo com algumas análises, um tipo diferente de composição simultânea é muito difundido em línguas de sinais. Os sinais em qualquer língua de sinais podem ser produzidos com uma mão ou com ambas as mãos. Sinais com duas mãos podem ser simétricos ou assimétricos, as duas mãos assumem a mesma configuração de mão e articulam o mesmo tipo de movimento no mesmo local (ou em locais espelhados em ou perto dos dois lados do corpo). Nos sinais assimétricos, uma mão (a mão preferida ou dominante) articula o sinal enquanto a outra mão (a mão não preferencial ou não dominante) é estática e funciona como local deste sinal.

11. Uma vez criadas, as composições estão sujeitas às pressões de mudança histórica. [...] Um elemento composto típico da ASL não consiste em dois morfemas intactos. Isso se dá por causa das mudanças estruturais que ocorrem como resultado da formação do elemento composto bem como devido a mudanças históricas posteriores à formação do composto. Assim, um sinal típico da ASL que chegou a nascer através das regras de formação de elementos compostos tecnicamente não é um elemento composto. Continuarei a descrevê-los como compostos mesmo 
assim, devido ao uso deste termo ser muito difundido e ao fato de não haver outro termo melhor.

\section{Referências}

ALVES, Ieda Maria. Neologismo: criação lexical. São Paulo: Ática, 1990.

BATTISON, Robbin. Lexical Borrowing in American Sign Language. Silver Spring, MD: Linstok Press, 1978.

BRENTARI, Diane. A Prosodic Model of Sign Language Phonology. Cambridge, MA: MIT Press, 1998.

CAPOVILLA, Fernando César. RAPHAEL, Walkiria Duarte. Dicionário Enciclopédico Ilustrado Trilíngue da Língua de Sinais Brasileira - Libras. São Paulo: Edusp, 2001. v. 1-2.

EGGINS, Suzanne. An Introduction to Systemic Functional Grammar. (2nd ed.). London/New York: Continuum, 2004.

FARIA-DO-NASCIMENTO, Sandra Patrícia. Representações Lexicais da Língua de Sinais Brasileira. Uma proposta lexicográfica. [Tese de doutorado] Brasília: Universidade de Brasília. Instituto de Letras. Departamento de Linguística, Português e Línguas Clássicas, 2009.

GIVÓN, Talmy. Functionalism and Grammar. Amsterdam: John Benjamins, 1995.

JAMESON, Lynn. et.al. ASL PRO. Disponível em < http://www.aslpro.com/ > . Acesso em 17 junho 2011. 
GUEDES, Vânia Lisboa da Silveira, MOLLICA, Maria Cecília de Magalhães, LEAL, Maria Beatriz Bezerra: Produtividade léxico-morfológica em artigos científicos em língua portuguesa numa perspectiva interdisciplinar. No prelo. Disponível em http://www.ppgci.ufrj.br/index. php/teste?download=167\%3Aprod utividade-lexico-morfologica-em-artigos-cientificos-em-lingua-portuguesa-numa-perspectiva-interdisciplinar

HOHENBERGER, Anette, HAPP, Daniela, LEUNINGER, Helen: Modality Dependent Aspects of Sign Language Production. In: Meier, R./Cormier, K. \& Quinto-Pozos, D. (orgs) Modality and Structure in Signed and Spoken Languages. Cambridge University Press: 2002, p. 112-142

KRIEGER, Maria da Graça. FINATTO, Maria José B. Introdução à Terminologia: teoria e prática. São Paulo: Contexto, 2004.

LIDDELL, Scott K. Grammar, Gesture, and Meaning in American Sign Language. Cambridge, UK: Cambridge University Press, 2003.

. JOHNSON, Robert E. American Sign Language: The Phonological Base. (1989) In: VALLI, Cleiton. LUCAS, Ceil. (Orgs.) Linguistics of American Sign Language: an introduction. Washington, D.C.: Clerc Books/Gallaudet University Press, 2000.

LIRA, Guilherme de Azambuja. SOUZA, Tanya Amara Felipe. Dicionário Digital da Lingua Brasileira de Sinais - Libras. Versão 2.0. Rio de Janeiro: 2005. Disponível em <www.ines.org.br $>$ e $<$ www.acessobrasil.org.br $>$. Acesso em 13 janeiro 2012.

MAX PLANCK INSTITUTE FOR PSYCHOLINGUISTICS. Sign Language Typology Group . In International Sign Communication - Workshop on "Cross-linguistic sign language research”. Nijmegen. The Netherlands. January 9-14, 2006

MEIR, Irit. ARONOFF, Mark. SANDLER, Wendy. PADDEN, Carol. "Sign languages and compounding” . In: SCALISE, Sergio and VOGEL, Irene (eds.). Cross-Disciplinary Issues in Compounding. John Benjamins Publishing Company, 2010. Disponível em < http://pages.ucsd.edu/ cpadden/files/Sign $\% 20$ Languages\%20and\%20Compounding.pdf > . Acesso em 27 setembro 2011. 
PFLAUM, Barbara. et.al. Needs Outreach - American Sign Language Library. Disponível em < http://www.needsoutreach.org/Pages/sl.html > Acesso em: 29 julho, 2011.

SAGI, Eyal, KAUFMANN, Stefan, CLARK, Brady. Semantic density analysis: comparing word meaning across time and phonetic space. In: GEMS '09 Proceedings of the Workshop on Geometrical Models of Natural Language Semantics, p. 104-111. Disponível em: http://dl.acm.org/ft_gateway.cfm?id=1705429\&typ $\mathrm{e}=$ pdf $\&$ CFID $=154514839 \&$ CFTOKEN $=15403735$

STUMPF, Marianne Rossi. Aprendizagem de Escrita de Língua de Sinais pelo Sistema Signwriting: Línguas de Sinais no papel e no computador. [tese de doutorado]. Porto Alegre: Universidade Federal do Rio Grande do Sul. Faculdade de Educação. Programa de pós-graduação em Informática na Educação, 2005.

SILVA, Eduardo Batista. BABINI, Mauricio. A preparação de material terminológico em língua inglesa por meio de ferramentas linguístico-computacionais. In: Trab. linguist. apl. vol.50 no.1 Campinas Jan./June 2011

SUTTON, Valerie. FROST, Adam. ISWA 2010 symbols. 2010. Disponível em $<$ http://www.signbank.org/iswa/cat_1.html > Acesso em 02 novembro 2012.

TENNANT, Richard A.; BROWN, Marianne Gluszak. The American Sign Language hand-shape Dictionary. Washington DC: Gallaudet University Press, 1998.

XAVIER, André N. Descrição Fonético-fonológica dos Sinais da Língua de Sinais Brasileira (LIBRAS). [Dissertação de Mestrado]. São Paulo: Universidade de São Paulo. Faculdade de Filosofia, Letras e Ciências Humanas. Programa de Pós-graduação em Semiótica e Linguística Geral, 2006.

Recebido em 12/12/2012

Aceito em 10/06/2013 